\title{
Sink-Independent Model in Wireless Sensor Networks
}

\author{
Sang-Sik Kim ${ }^{1}$, Kwang-Ryul Jung ${ }^{1}$, Ki-Il Kim ${ }^{2, *}$, and Ae-Soon Park ${ }^{1}$ \\ ${ }^{1}$ Electronics and Telecommunications Research Institute, \\ 2 Department of Information Science, Research Institute of computer and Information \\ Science, GyeongSang National University, 900 Gajwa-dong, Jinju, 660-701, Korea \\ \{pstring, krjung\}@etri.re.kr, kikim93@gmail.com, \\ aspark@etri.re.kr
}

\begin{abstract}
Wireless sensor networks generally have three kinds of objects: sensor nodes, sinks, and users that send queries and receive data via the sinks. In addition, the user and the sinks are mostly connected to each other by infrastructure networks. The users, however, should receive the data from the sinks through multi-hop communications between disseminating sensor nodes if such users move into the sensor networks without infrastructure networks. To support mobile users, previous work has studied various user mobility models. Nevertheless, such approaches are not compatible with the existing routing algorithms, and it is difficult for the mobile users to gather data efficiently from sensor nodes due to their mobility. To improve the shortcomings, we propose a view of mobility for wireless sensor networks and propose a model to support a user mobility that is independent of sinks.
\end{abstract}

Keywords: User Mobility Support, Wireless Sensor Networks.

\section{Introduction}

Wireless sensor networks typically consist of three objects: user, sink, and sensor node [1]. Firstly, a user is an object that disseminates an interest in the sensor field and collects data about the interest from sensor nodes. Secondly, a sink is an object that collects data. The sink receives an interest from a user and disseminates the interest inside sensor fields. The sink receives sensing data from sensor nodes and forwards the sensing data to the user. Lastly, a sensor node is an object that generates data about the interest and delivers the data to a sink.

The user and the sinks are mostly connected to each other by infrastructure networks. The users, however, should receive the data from the sinks through multihop communications between sensor nodes if such users move around the sensor networks without infrastructure networks. Recently, applications transmitting data to moving users inside sensor fields, such as rescue in a disaster area or maneuvers in a war zone, have been on the rise in large-scale sensor networks [5]. (Firefighters and soldiers are users gathering data from sensor networks.)

${ }^{*}$ Corresponding author. 


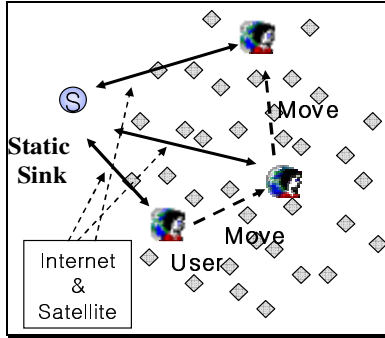

Fig. 1. Direct user-network communication model

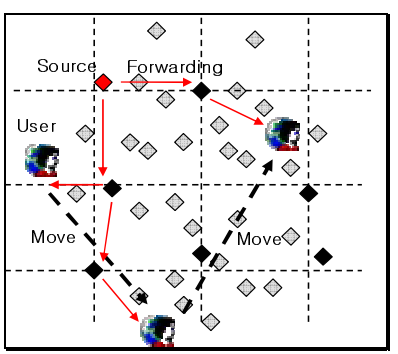

Fig. 2. GPS based usernetwork communication model

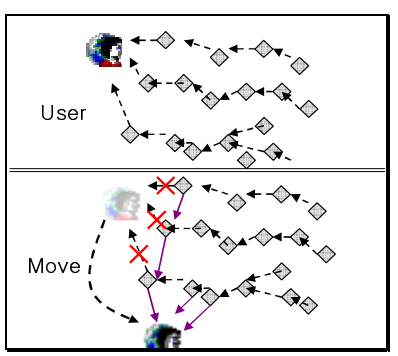

Fig. 3. Topology control based user- network communication model

To support mobile users in wireless sensor network, previous work has studied various user mobility models. But, until now, only three models supported the mobility of users for those applications: the direct user-network communication model, the GPS-based user-network communication model, and the topology-controlbased user-network communication model.

The direct user-network communication model (D-COM) is shown in Fig. 1. It supports the mobility of a user on the assumption that the user communicates directly with sinks through infrastructure networks, namely, the Internet, such as communication systems in traditional sensor networks [1]. And, users can communicate directly with the networks via the sinks. But, in applications such as rescues in a disaster area or maneuvers in a war zone, circumstances without infrastructure networks, except sensor networks, are more prevalent. Hence, the assumption that a user and a sink can communicate directly is not entirely accurate.

The GPS-based user-network communication model (G-COM) is seen in Fig. 2. G-COM is source-based topology [5], [6], [7]. In G-COM, sensor nodes proactively construct a GRID system with GPS receivers. G-COM assumes that all sensor nodes have their own GPS receivers and an ability that constructs a GRID. A sensor node, i.e. source, with a stimulus is going to make a GRID in a sensor field. Once a GRID is set up, mobile user floods its interests within a cell only where the user is located. When a sensor node on a GRID receives interests, it sends interests to the source along a GRID path and data from the source are forwarded to the user.

The topology-control-based user-network communication model (T-COM) is seen in Fig. 3. This model supports the mobility of the user by reflecting the movement of the user [8], [9]. In T-COM, the user and sensor nodes construct a tree that is rooted at the user. The user always maintains the tree and gathers data. Intuitively, G-COM and T-COM seem to be suitable for the aforementioned applications. But, these models also have various problems. First of all, they cannot use existing effective data collection algorithms [2], [3], [4] between a sink and sensor nodes because of low protocol compatibility. Accordingly, such algorithms can hardly be exploited if users in sensor networks have mobility. The other problem is that the cost of the overhead to reorganize the network topology and reconstruct dissemination paths is expensive. In G-COM, all sensor nodes make the topology based on location information. Accordingly, each sensor node must have its own GPS receiver. The cost 
Table 1. Taxonomy of Mobility Type

\begin{tabular}{ccccccc}
\hline $\begin{array}{c}\text { Mobility } \\
\text { Type }\end{array}$ & $\begin{array}{c}\text { Compatibility } \\
\text { with Existing } \\
\text { Static Sink } \\
\text { Routing Protocols }\end{array}$ & Feasibility & $\begin{array}{c}\text { GPS } \\
\text { receivers } \\
\text { for sensors }\end{array}$ & $\begin{array}{c}\text { Control } \\
\text { Overheads } \\
\text { according to } \\
\text { user mobility }\end{array}$ & $\begin{array}{c}\text { Control } \\
\text { Overheads to } \\
\text { support } \\
\text { multiple users }\end{array}$ & $\begin{array}{c}\text { Help of } \\
\text { infrastructure } \\
\text { networks }\end{array}$ \\
\hline D-COM & High & Low & Needless & Low & Low & Mandatory \\
G-COM & Low & Middle & Mandatory & Middle & Low & Needless \\
T-COM & Low & High & Needless & High & High & Needless \\
A-COM & High & High & Needless & Low & Low & Needless \\
\hline
\end{tabular}

of GPS receivers is decreasing, but the overall cost is still high. In T-COM, similarly, user mobility causes topology reconstruction. Users in T-COM have a tree that is rooted at each mobile user. If users move into a new location, then the root of trees must be changed, as seen in Fig. 3. This leads to enormous overhead to sensor nodes.

Hence, this paper proposes a novel agent-based user-network communication model (A-COM). A-COM collects data through a temporary agent and delivers the data to mobile users. In A-COM, the user appoints a sensor node to act as an agent, and the agent forwards interests to the sink. The sink collects data from sensor nodes using the existing data collection algorithm in static sink sensor networks [2], [3], [4]. The collected data are finally forwarded to the user. (If there is no sink, the agent directly disseminates interests and collects data.) A-COM has various advantages, as can be seen in Table 1. First of all, A-COM has the compatibility with existing static sink routing protocols without infrastructure networks. In addition, the users in ACOM do not make a topology (tree or GRID) and communicate only with agents. So, the users are free from topology control. The user's freedom saves energy and enables more users to participate in this model even if the sensors have no GPS receivers.

\section{Model Analysis}

In our model, if a user intends to obtain data while moving, the user appoints a sensor to act as an agent and forwards an interest to the agent. If there is one or more $\operatorname{sink}(\mathrm{s})$, the agent forwards interests to sensor networks via sink(s). The number of sinks, however, depends on the network policy. A network administrator might want to set a single or more sinks in the sensor field, or alternatively the sensor field may be hazardous as he cannot reach the field. Hence, we consider three scenarios according to the number of sinks and describe the scenarios based on following assumptions.

- A user can communicate with static sinks only through sensors because networks within sensor fields are infrastructure-less networks. In addition, It is possible that multiple sinks are deployed in sensor networks and are connected to each other.

- The data which one sink collects is aggregated by the sinks. The aggregated data is shared by every multiple static sinks through the infrastructure network.

- The interest describes how many times the sink forwards the gathered data set. 


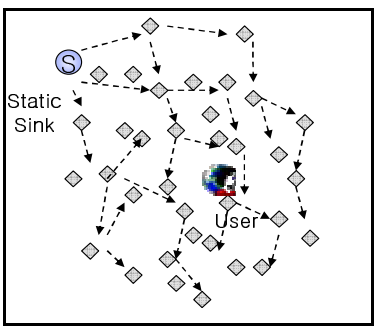

Fig. 4. Dissemination of Sink Announcement Message

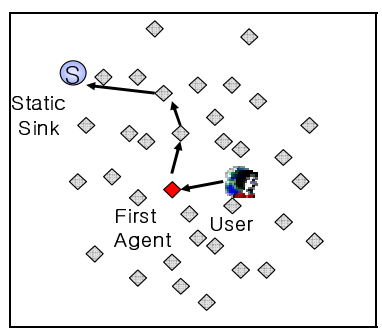

Fig. 5. Interest Dissemination of the user

\subsection{Scenario 1: Sensor Fields with Only One Sink}

Dissemination of sink announcement message and user interest. In the initial stage of the sensor network, a sink floods a sink announcement message to announce itself inside the whole sensor field (Fig. 4). As a result of the flooding announcement message, every sensor node knows the hop counts and next hop neighbor sensor node. While moving inside the sensor fields, the user selects the nearest node as a first agent, as shown in Fig. 5. The user delivers an interest to the first agent. The first agent forwards the interest to the next hop neighbor node toward the sink. The next hop sensor node, which has had the interest delivered to it, also forwards the interest to the next hop neighbor node toward the sink. This process continues until the sink receives the interest of the user. Also, a route for the interest from the sink to the user has been established through this process. The established route vanishes from the network when the described period in the interest is over.

Data collection. A sensor network with a static sink is a network where sensing data from sensor nodes should be transmitted to the static sink through multi-hop communication. So, existing routing algorithms [2], [3], [4] for a static sink can be used (e.g., routing algorithms collecting data by periods, routing algorithms collecting a minority event, or routing algorithms detecting a moving object.) In Fig. 6, the static sink can forward interests from users to sensors and gather data from sensor networks according to the existing routing protocols. If all data are gathered by routing protocols, the static sink aggregates all data and forwards an aggregated data to the first agent. A user may move to another place after sending an interest to the first agent. In this case, the user selects another agent that can communicate with the first agent. Also, the user makes a new connection between the newly selected agent and the original agent. These agents are used for forwarding the aggregated data.

\subsection{Scenario 2: Sensor Fields with Multiple Sinks}

Basically, the difference between Scenario 1 and Scenario 2 is only the number of sinks. If there are more than one sink in the sensor field, this means a separation of the sensor fields. As a result of sink announcement message dissemination in this case, all 


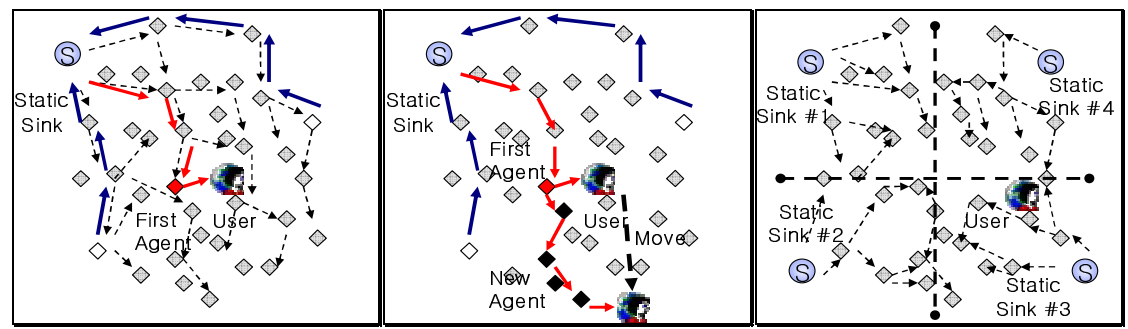

Fig. 6. Data Propagation to the user

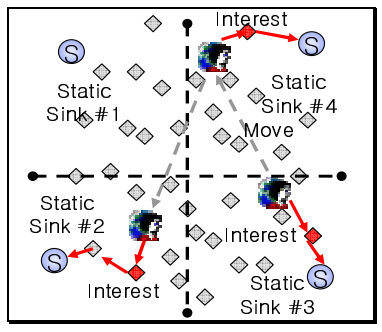

Fig. 9. Interest Dissemination of the user with multiple sinks
Fig. 7. Mobility support of the user
Fig. 8. Seperation of the Sensor Fields

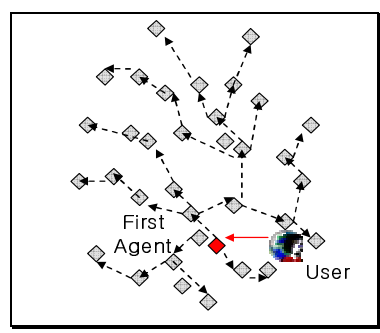

Fig. 10. First Agent Selection and Announcement

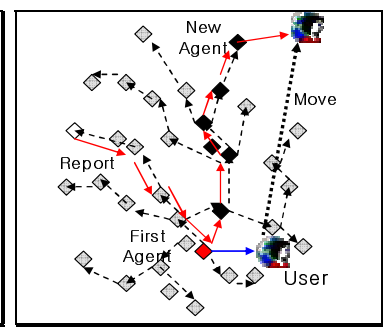

Fig. 11. Mobility support of the user

sensor nodes know the nearest sink according to the hop counts. Accordingly, Interest dissemination of the user targets the nearest sink from the agent, as shown Fig 8 . The targeted sinks can be changed whenever the user wants to send its interests (see Fig. 9). Nevertheless, Mobility support of the user and data propagation of the sink is still the same with Scenario 1. In addition, users may not be able to recognize how many static sinks are in the sensor fields. This means that the proposed model is independent of the number of sinks. A user can receive the data from the nearest sink to its position. Short hops communications between a user and a sink are possible. This saves energy, enhances the data delivery ratio, and reduces delay.

\subsection{Scenario 3: Sensor Fields with No Sink}

The sensor fields without a sink are a special type of the sensor networks. If the sensor field is hazardous as network administrator cannot reach the field (ex. Battlefield), it may not have any sinks. Because there is no sink in the sensor field, the sensor network cannot practice sink announcement message dissemination process for itself. In this case, users must appoint the nearest sensor node as first agent, and the first agent disseminates sink announcement message. As shown Fig 10 and 11, users examine nearby sensor nodes whether there is a sink in the sensor field or not. If not, users appoint the nearest sensor node to first agent. Once a sensor node becomes first agent, it acts like the sink of Scenario 1. Hence, other processes such as sink announcement message dissemination and data propagation of the sink are the same as Scenario 1. The first agents must return to the original state after the described 
period. This means that the first agents are appointed whenever users want to send its interest. Then, the first agents are reactively selected and practice all process for user mobility. In the whole network, therefore, the sensor network can remain in an idle state in case there is no user in the sensor field. This is a positive effect because there is no control messages and interests in the network.

\section{Performance Evaluation}

We evaluate the proposed model in Qualnet, a network simulator [12]. A sensor node's transmitting and receiving power consumption rate are $0.66 \mathrm{~W}$ and $0.39 \mathrm{~W}$. The transceiver in the simulation has a $50 \mathrm{~m}$ radio range in an outdoor area. The sensor network consists of 100 sensor nodes, which are randomly deployed in a $300 \mathrm{~m} \mathrm{x}$ $300 \mathrm{~m}$ field. And the user which follows a random waypoint model of $10 \mathrm{~m} / \mathrm{s}$ speed and 10 second pause time moves into the sensor field. The user disseminates an interest at an interval of every 10 seconds. The simulation lasts for 500 seconds.

\subsection{Impact of the Number of Static Sinks}

Scenarios 1 and 2 of A-COM can be compared with the D-COM because G-COM and T-COM have no static sink. We first study the impact of the number of sinks on ACOM's performance. The number of sinks varies from 1, 2, 3, 4 to 5. And there is only one user in the sensor field. In this part, we compare Scenarios 1 and 2 to DCOM regarding lifetime, delay, and delivery ratio. Fig. 12 shows the number of interest rounds, namely, network lifetime. The number of interest rounds shows little difference between A-COM and D-COM. This means that A-COM can manage sensor fields as well as D-COM without infrastructure. In addition, the lifetime is increased according to the number of sinks. This is a side effect of multiple sinks. Sinks separate the sensor field, and besides, users only use the nearest sink to send interests and receive replies. Users can use the shortest path to communicate with multiple sinks. As a result of the shortest communication, the lifetime in A-COM is enhanced according to the number of sinks. The delay is also enhanced by this side effect of multiple sinks. A-COM basically has some delay due to multi-hop communication between users and sinks. However, the delay is diminished according to the number of sinks, as shown in Fig. 13. Nevertheless, the data delivery ratio of ACOM is comparable with D-COM, as shown in Fig. 14. This also proves that the proposed model can manage sensor fields without infrastructure.

\subsection{Impact of the Number of Users}

The number of users only results in path increase between users and sinks. D-COM uses direct communication between users and sinks, and A-COM uses multi-hop communication. A-COM has more paths and consumes more energy. (e.g., five users in A-COM consumes five times of the energy that is consumed by one user.) However, it is a tradeoff between energy and infrastructure. Although A-COM has more energy consumption and delays than D-COM, the merit of A-COM is infrastructure-less communication systems. 


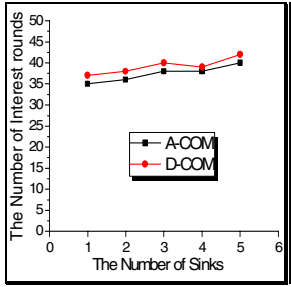

Fig. 12. Network lifetime for the Number of Sinks

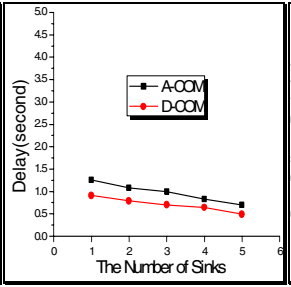

Fig. 13. Delay for the Number of Sinks

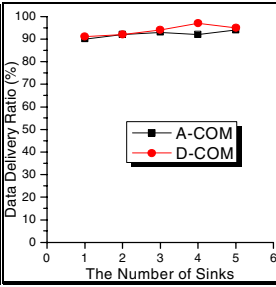

Fig. 14. Data Delivery Ratio for the Number of Sinks

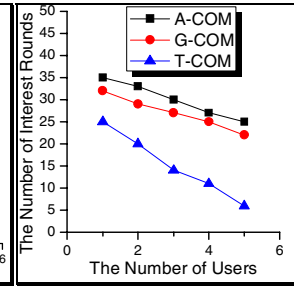

Fig. 15. Network Lifetime for the Number of Users

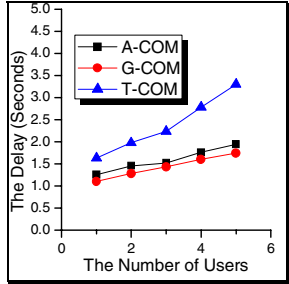

Fig. 16. Delay for the number of Users

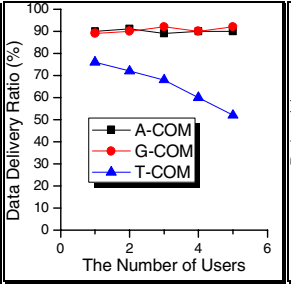

Fig. 17. Data Delivery Ratio for the Number of Users

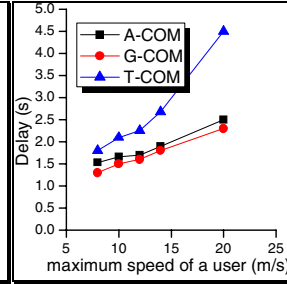

Fig. 18. Delay for User Speed

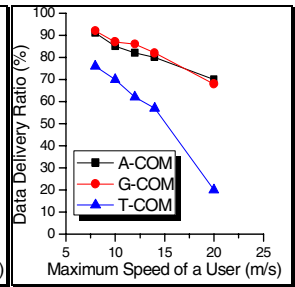

Fig. 19. Data Delivery Ratio for User Speed

Scenario 3 of A-COM can be compared with G-COM and T-COM because Scenario 3 of A-COM, G-COM, and T-COM have no static sinks. There are no sinks, and the number of users varies from 1, 2, 3, 4 to 5. In this part, we compare Scenario 3 of A-COM to G-COM and T-COM regarding lifetime, delay, and delivery ratio. G-COM and T-COM make and change the topology proactively, but Scenario 3 of A-COM reactively makes and shares it among users. Generally, users move about the sensor field only and generate its interest occasionally. Hence, sensors in Scenario 3 can save considerable energy. Alternatively, sensors in G-COM and T-COM maintain a topology continuously. Fig. 15 shows each lifetime of these sensor networks. As shown in Fig. 15, the lifetime of T-COM is considerably low due to frequent topology change and that of G-COM is relatively low due to GRID maintenance.

In Fig. 16, G-COM has little delay due to proactive GRID topology by the GPS receiver. T-COM proactively creates the topology, but frequent topology changes of T-COM delay data delivery considerably. The delay of Scenario 3, as shown in Fig. 16, however, is only a little high due to the reactive first agent selection and topology construction. In the case of the data delivery ratio, A-COM and G-COM in Fig. 17 are similar except for T-COM. The reason is frequent topology change. Topology change messages disturb the data delivery ratio.

\subsection{Impact of the User Mobility}

We lastly evaluate the impact of user speed on A-COM. We vary the maximum speed of a user from $8,10,12,14$ to $20 \mathrm{~m} / \mathrm{s}$. We assume that there is one user in the sensor field. In this part, we compare Scenario 3 to G-COM and T-COM because D-COM is independent of user speed. Fig. 18 shows the delay in data delivery, which slightly 
increases as the user moves faster. The delay depends on a movement operation that is processed by the user. The faster a user moves, the more the time is needed to establish a connection between the user and the network. Nevertheless, the delay of A-COM is comparable with G-COM because A-COM creates only one communication path between the user and its first agent. The delay of T-COM, on the other hand, is relatively higher than the others due to frequent topology changes.

And, Fig. 19 shows the data delivery ratio when the user's moving speed changes. The data delivery ratio of A-COM is slightly decreased according to the delay. But the data delivery ratio remains around $0.7-0.9$; nevertheless, the user moves faster. Besides, the data delivery ratio of G-COM remains high because the GPS receiver may help the user with geographical routing. On the other hand, the data delivery ratio of T-COM is relatively lower than the others because it has too many topology changes when moving. The results in Fig. 18 and Fig 19 mean that A-COM is fast and stable without GPS receiver.

\section{Conclusion}

In this paper, we propose a novel agent-based user-network communication model to support the mobility of users in wireless sensor networks. In the proposed network model, the user can receive data with a higher data delivery ratio and in a faster time without infrastructure. We verified that the lifetime of sensor networks is prolonged because the reactive path construction decreases the energy consumption of sensor nodes. Also, we verified that performance of the data delivery ratio and the delay never falls; nevertheless, communication between the user and the network for guaranteeing movement of the user is supported by only sensor nodes.

\section{References}

1. I.F. Akyildiz, et al., "A survey on sensor networks," Communications Magazine, Aug. 2002.

2. C. Intanagonwiwat, et al., "Directed diffusion: A scalable and robust communication paradigm for sensor networks," ACM Mobicom, 2000.

3. C. Schurgers, et al., "Energy efficient routing in wireless sensor networks," MILCOM 2001.

4. W.R. Heinzelman, et al., "Adaptive Protocols for Information Dissemination in Wireless Sensor Networks," ACM Mobicom, 1999.

5. F. Ye, et al., "A Two-Tier Data Dissemination Model for Large-scale Wireless Sensor Networks," ACM MobiCOM, Sept. 2002.

6. S. Kim, et al., "SAFE: A Data Dissemination Protocol for Periodic Updates in Sensor Networks," Distributed Computing Systems Workshops 2003.

7. H. L. Xuan and S. Lee, "A Coordination-based Data Dissemination Protocol for Wireless Sensor Networks," IEEE ISSNIP, Dec. 2004.

8. K. Hwang, et al., "Dynamic sink oriented tree algorithm for efficient target tracking of multiple mobile sink users in wide sensor field," IEEE VTC, Sep. 2004.

9. S. R. Gandham, et al., "Energy Efficient Schemes for Wireless Sensor Networks with Multiple Mobile Base Stations," IEEE GLOBECOM, Dec. 2003.

10. Scalable Network Technologies, Qualnet, available: http://www.scalable-networks.com. 